\title{
An automated Y-maze based on a reduced instruction set computer (RISC) microcontroller for the assessment of continuous spontaneous alternation in rats
}

\author{
Francisco J. Heredia-López ${ }^{1}$ Fernando J. Álvarez-Cervera ${ }^{1}$ - José G. Collí-Alfaro ${ }^{1}$. \\ José L. Bata-García ${ }^{1}$ • Gloria Arankowsky-Sandoval ${ }^{1}$ • José L. Góngora-Alfaro ${ }^{1}$
}

Published online: 12 November 2015

(C) Psychonomic Society, Inc. 2015

\begin{abstract}
Continuous spontaneous alternation behavior $(\mathrm{SAB})$ in a Y-maze is used for evaluating working memory in rodents. Here, the design of an automated Y-maze equipped with three infrared optocouplers per arm, and commanded by a reduced instruction set computer (RISC) microcontroller is described. The software was devised for recording only true entries and exits to the arms. Experimental settings are programmed via a keyboard with three buttons and a display. The sequence of arm entries and the time spent in each arm and the neutral zone (NZ) are saved as a text file in a non-volatile memory for later transfer to a USB flash memory. Data files are analyzed with a program developed under LabVIEW® environment, and the results are exported to an Excel ${ }^{\circledR}$ spreadsheet file. Variables measured are: latency to exit the starting arm, sequence and number of arm entries, number of alternations, alternation percentage, and cumulative times spent in each arm and NZ. The automated Y-maze accurately detected the SAB decrease produced in rats by the muscarinic antagonist trihexyphenidyl, and its reversal by caffeine, having $100 \%$ concordance with the alternation percentages calculated by two trained observers who independently watched videos of the same experiments. Although the values of time spent in the arms and NZ measured by the automated system had small discrepancies with those calculated by the observers, Bland-Altman analysis showed $95 \%$ concordance in three pairs of comparisons, while in one it was $90 \%$,
\end{abstract}

José L. Góngora-Alfaro

jlgongoralf@gmail.com

1 Departamento de Neurociencias, Centro de Investigaciones Regionales “Dr. Hideyo Noguchi”, Universidad Autónoma de Yucatán, Avenida Itzáes No. 490 x 59, Mérida, Yucatán, México 97000 indicating that this system is a reliable and inexpensive alternative for the study of continuous SAB in rodents.

Keywords Behavioral tests $\cdot$ Locomotion $\cdot$ Short-term memory $\cdot$ Infrared sensor $\cdot$ Automated system ·

Anticholinergics

\section{Introduction}

The spontaneous alternation test relies on the innate rodent behavior of alternating unrewarded visits to the arms of a maze in the shape of a T or a Y (Hughes, 2004; Lalonde, 2002). This test was originally devised for the T-maze, for which either a free- or a forced-trial paradigm has been used. In the free-trial procedure the animal is placed at the end of one arm, and is allowed to freely choose to enter one of the two other arms, after which it is withdrawn and placed again in the starting arm for the next trial (Lalonde, 2002; Morellini, 2013). In the forced-trial test the two arms that are not used as the starting place have doors that can block the entrance to either of them. In the first trial one of the doors is closed, and then the rodent is allowed to explore the maze. Once it has entered the open arm, it is removed and returned to the starting point for the second trial, in which the arm that was previously closed is now open (Lalonde, 2002). This usually gives rise to a stronger spontaneous alternation behavior (SAB), since rodents have a natural tendency to explore unfamiliar spaces. Thus, when they face the option of entering two arms they usually choose the one that has not been recently visited (Morellini, 2013). Given that SAB relies on the ability of the animal to remember which arm was visited less recently, the test is conceived as a method for the experimental assessment of working memory, which is a form of short-term memory, involving attention and information processing, and is 
devoted to the planning and execution of an ongoing behavior (Hughes, 2004; Morellini, 2013).

Although valuable, the free- and forced-trials in the T-maze have the limitation that the frequent handling of animals inevitably causes stress-induced fear that influences their alternation behavior (Hughes, 2004). This has led to the design of another paradigm known as continuous $\mathrm{SAB}$ in which animals freely explore the arms of a Y-maze for several minutes, without repeated manipulation by the experimenter (Hughes, 2004; Morellini, 2013). Since continuous SAB is a simple test that causes minimum stress to animals and does not require any gratifying reward, it has gained popularity among scientists to assess the impact of drugs on working memory (Hughes, 2004). It has the additional advantage of providing a measure of the locomotor activity of rodents by counting the number of entries to the maze arms (Hughes, 2004).

In the most common procedure for the measurement of continuous $\mathrm{SAB}$, an observer records the sequence of entries of a rodent into the arms of a Y-maze over 5-8 min (Drew et al., 1973; Fraser et al., 1997; Hefco et al., 2003; Hooper et al., 1996; Krebs-Kraft \& Parent, 2008; Lelong et al., 2003; Stefani et al., 1999; Stone et al., 1992; Yamada et al., 1996). However, although simple and inexpensive, this method has several limitations. For instance, the presence of the observer may alter the rodent behavior in the Y-maze, yielding spurious results. Likewise, during the execution of repeated tests, the observer might miss valuable information due to fatigue or distractions. Additionally, at a later stage involuntary alterations may arise as a result of transcription errors. Though there is the alternative of filming each trial, the task of watching the videos in order to count the sequence of entries to the arms and the duration of each visit is time-consuming and may not be error-free. Furthermore, the data analysis used to calculate the percentage of spontaneous alternation, time spent in arms, etc., is another potential source of errors. All these limitations could be circumvented by means of automated devices capable of recording and analyzing the sequence of continuous spontaneous alternations performed by rodents in the Y-maze. However, studies reporting on automated systems for the study of continuous SAB are scarce.

Sarter et al. (1988) were pioneers in publishing a brief outline of an automated Y-maze capable of recording the sequence in which a mouse visited the three arms through the successive interruption of two infrared beams, located at 1 and $14 \mathrm{~cm}$ from the entrance of each arm, respectively. However, these authors did not provide details about the hardware or software, nor did they demonstrate the efficacy and accuracy of their automated system. More recently, Lennartz (2008) evaluated the continuous $\mathrm{SAB}$ of rats in a plus-maze which had arms equipped with three infrared beams, two near the entrance and one at midpoint of the arm's length. The author noted that occasionally the system recorded a false double entry (two consecutive entries into the same arm), which occurred when the rat interrupted the midpoint beam, stepped toward the central area without leaving the arm, and then turned around and broke the midpoint beam again (Lennartz, 2008). Using a different approach, Detrait et al. (2010) used a commercial video-tracking system (EthoVision XT®) for the implementation and validation of an automated scoring procedure for continuous SAB testing of mice in a Y-maze. System optimization consisted in redefining the central maze area to avoid counting false arm entries, and incorporating an analysis program to calculate the alternation percentage (Detrait et al., 2010).

The commonly used criterion to define that an animal has performed an arm entry is when it places all four paws within the boundaries of the arm (Detrait et al., 2010; Fraser et al., 1997; Hefco et al., 2003; Hooper et al., 1996; Lelong et al., 2003; Lennartz, 2008; Sarter et al., 1988; Yamada et al., 1996), even though its tail could remain within the small triangular space where the three arms converge. Although simple for an observer to follow, this norm is subject to errors when using automated devices. This is clearly illustrated by the results of a study aimed at assessing the reliability of a video-tracking system for scoring continuous $\mathrm{SAB}$ in mice, using software initially adjusted to count an "arm exploration ... as entering at least the first third of an arm" (Detrait et al., 2010). When comparing the data recorded by the videotracking system with those registered by an observer, the authors found an "average $20 \%$ variation between the two scoring techniques", which they ascribed to the fact that the "normal rodent behavior consisted of repeatedly going around within the central zone of the Y-maze," thus fooling the system which "considered any animal located in this area as exploring the arm, while it was not" (Detrait et al., 2010). However, after redefining an arm exploration as the presence of the animal in its middle or distal thirds, the automated analysis had $100 \%$ concordance with the observer (Detrait et al., 2010).

With the aim of providing an improved alternative for the automated recording and analysis of continuous SAB in rodents, here we describe in detail the design of a Y-maze equipped with nine infrared beams controlled by a reduced instruction set computer (RISC) microcontroller. The software governing the RISC was devised to count an arm entry only when the rat moves at least into the middle third of the arm's length, thus ensuring that the system only records true arm entrances (Detrait et al., 2010). Some important features are: (1) the experimental settings can be programmed via a keyboard with only three buttons and a display, (2) the sequence of arm entries and the time spent in each arm and the central zone are saved in a non-volatile memory for later transfer as a compact text file to a conventional USB flash memory, (3) single files or sets of them can be analyzed with a program developed under the LabVIEW® environment (National Instruments Corp., Austin, TX, USA) and the results are 
exported to a spreadsheet file, (4) the system is unaffected by insufficient contrast between the rat and the background, nor by lighting conditions, as could be the case with some videobased methods (Bailoo et al., 2010), and (5) it has the great advantage of using very low cost electronic components for the automation of the Y-maze; this cost is comparable to that of a general purpose equipment designed to monitor locomotion and various types of behaviors in rodents, and which is also based on infrared optocouplers (McLelland et al., 2015).

\section{Materials and methods}

The Y-maze we describe is made of black acrylic, and its diagram is shown in Fig. 1. The far end of arm A is conventionally the starting point for the SAB test. The sides of this arm are both $46 \mathrm{~cm}$ in length, in contrast with the lengths of arms B and C, which are also $46 \mathrm{~cm}$ on the external side (top of the figure) but only $42 \mathrm{~cm}$ on the inner side (middle part of the figure) due to the slanted Y-shaped design. The width of all three arms is $14.6 \mathrm{~cm}$. The angle formed between arms B and $\mathrm{C}$ is $156^{\circ}$, whereas the angle between $\mathrm{A}$ and $\mathrm{B}$, or between $\mathrm{A}$ and $\mathrm{C}$, is $102^{\circ}$. The three arms converge in a triangular area designated as the neutral zone (NZ).

The detection of the position of the rat within the maze is achieved by using infrared light sources (emitters) and detectors (receivers), placed transversally on opposite sides of each arm (Fig. 1). This emitter-detector pair is known as an optocoupler (OC), which in this case is formed by an IR383 infrared emitting diode (blue lens, $\phi=5 \mathrm{~mm}$ ) and a PT1302B/ $\mathrm{C} 2$ phototransistor (black lens, $\phi=5 \mathrm{~mm}$ ) (Steren, México). Though the lenses of the emitter and the detector protrude about $4 \mathrm{~mm}$ towards the maze interior, the rats have never gnawed the lenses, albeit they occasionally spend a few seconds $(\leq 5)$ sniffing at them. When the rat's body interrupts the infrared light beam generated by the emitter, a signal is produced in the corresponding receiver that is associated with the position of the given sensor and, hence, of the rat. Each arm of the Y-maze is equipped with three OCs, whose positions are depicted in the diagram in Fig. 1. The system includes the associated circuits and firmware (described below) designed to validate and record the sequence of entrances to the arms and the NZ, as well as their durations.

All emitters and receivers were located $5 \mathrm{~cm}$ above the maze floor, a height that proved to be adequate for the size of adult rats. The locations of the OCs were defined on the basis of the approximate body length of adult rats ( $\geq 60$ days old), usually measured from nose to anus, which varies according to age and weight. Thus, Pullen (1976) reported that Wistar rats of both sexes at the age of 75 days weighed $182 \pm$ $15 \mathrm{~g}$ and had an average body length of $18.2 \pm 8.0 \mathrm{~cm}(\mathrm{SD}, \mathrm{n}=$ 12). On the other hand, McCay et al. (1935) reported that male albino rats at the age of $359 \pm 25$ days weighed $439 \pm 10 \mathrm{~g}$ and had a mean body length of $21.9 \mathrm{~cm}(\mathrm{n}=12)$. Using three OCs per arm insured that the spatial detection of the animal in all areas of the maze was reliable. Hence, taking as a reference the point where each arm joins the NZ, the proximal OC (closest to the arm entrance) was arbitrarily positioned at $3.5 \mathrm{~cm}$ from

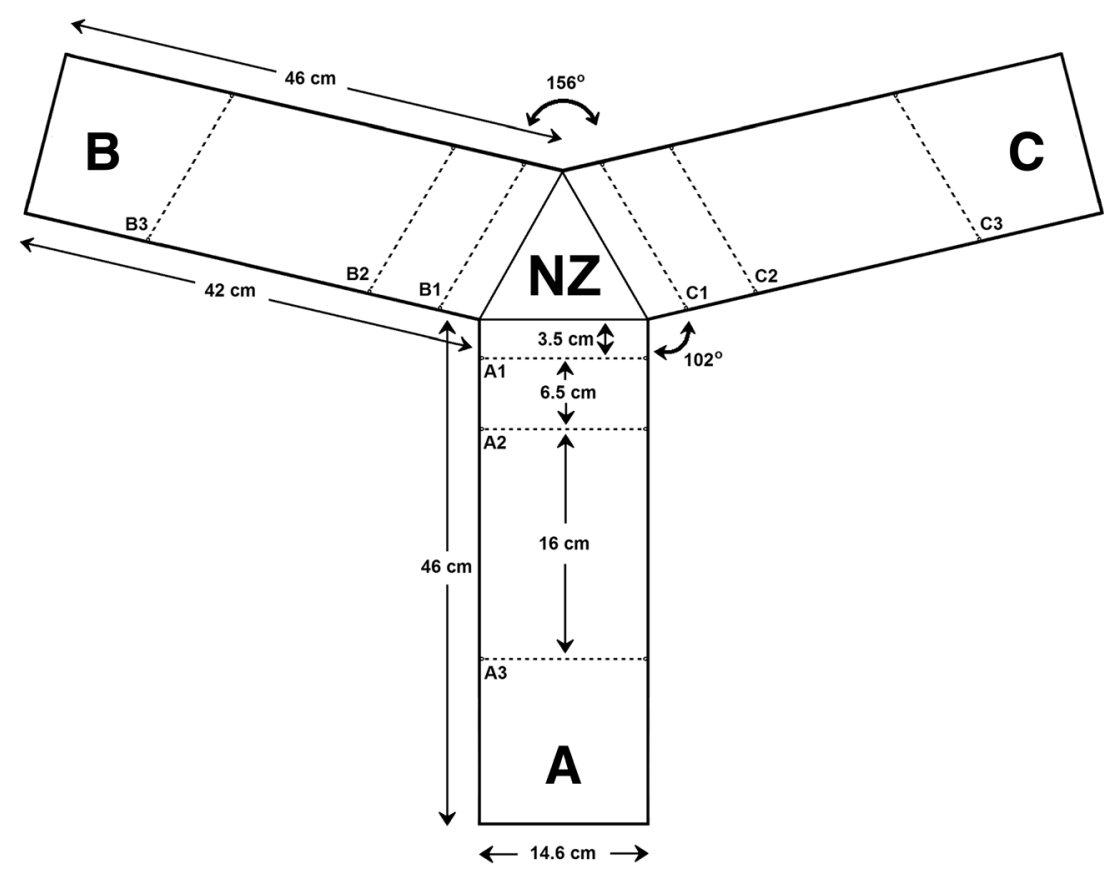

Fig. 1 Diagrammatic representation of the automated Y-maze (top view). For arm A optocouplers (OCs) are identified as follows: A1 refers to the proximal OC, nearest to the neutral zone (NZ); A2 is the medial OC; and
A3 the distal OC, farther away from the NZ. Similar coding is used for the OCs in arms B and C. Dotted lines represent infrared beams 
the NZ in order to detect the initial incursion of the rat to an arm, thus starting the OC interruption sequence. With the purpose of detecting that the rat was advancing into the arm, the medial OC was placed at $10 \mathrm{~cm}$ from the NZ, which is over one-third of the body length of an adult rat (McCay et al., 1935; Pullen, 1976). Considering that the total length of an adult rat is several centimeters longer than the distance between the front and rear paws when the animal is on all fours, the distal $\mathrm{OC}$ was placed $16 \mathrm{~cm}$ beyond the medial sensor, for a total distance of $26 \mathrm{~cm}$ from the NZ. This separation insured that whenever a rat interrupted the infrared beam from the distal OC, its four paws were necessarily located within the space of the arm being explored. Finally, according to the programmed OC interruption sequence, in order to validate occluding the proximal $\mathrm{OC}$, while continuing to block the distal sensor. That is, at this point the part of the rat that was innermost in the arm would be more than $26 \mathrm{~cm}$ away from the NZ.

With this spacing of the OCs the system can discriminate between a true and a false arm entry. A true arm entry was recorded once the rat reached a position past the proximal third of each arm (i.e., at least $26 \mathrm{~cm}$ from the NZ, where the distal OC is located), a situation in which all four paws the visit to the arm it was necessary that the rat stopped

are necessarily placed inside of it, and which is the standard criterion for an arm entry (Detrait et al., 2010; Fraser et al., 1997; Hefco et al., 2003; Hooper et al., 1996; Lelong et al., 2003; Lennartz, 2008; Sarter et al., 1988; Yamada et al., 1996). Entries to an arm not meeting this condition were considered false events (Detrait et al., 2010) and, thus, not counted. A particular condition arises when a rat leaves an arm by going into the NZ and then re-enters the same arm (without first visiting any of the other two arms). This case of two (or more) consecutive visits to the same arm is considered as a single visit (a re-entry), and the durations of the two (or more) entries are added. However, the duration of the intermediate visit(s) to the NZ is subtracted to give the final duration of the arm entry.

In Fig. 2 each OC is identified by a letter corresponding to the arm, and a number indicating the position ( $1=$ proximal; $2=$ medial, and $3=$ distal). By convention, experiments start by placing the rat in the distal portion of arm A, with its head facing away from the NZ. Therefore, in this automated maze the recording of events begins when the light beam of the distal OC in this arm, labeled as A3, is interrupted. This signal starts the electronic stopwatches for the previously programmed experimentation time, as well as for the time initially spent by the rat in arm A.

\section{e}

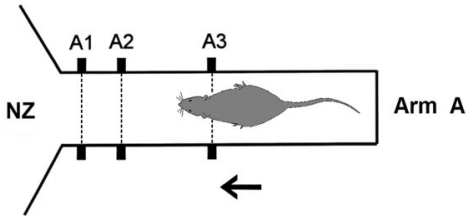

b

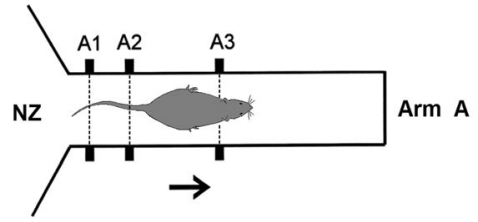

C

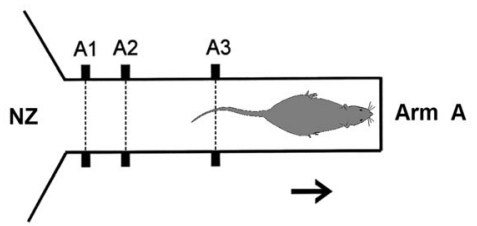

d

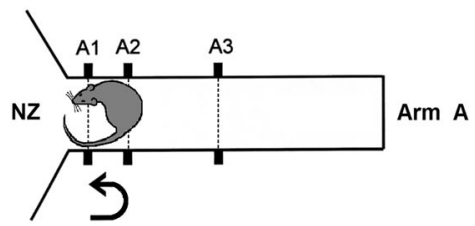

Fig. 2 Sequence of events for a valid entry into or an exit from an arm of the automated Y-maze. Drawings (a) to (c) illustrate the sequence of optocoupler interruptions that represent a true arm entrance. Drawing
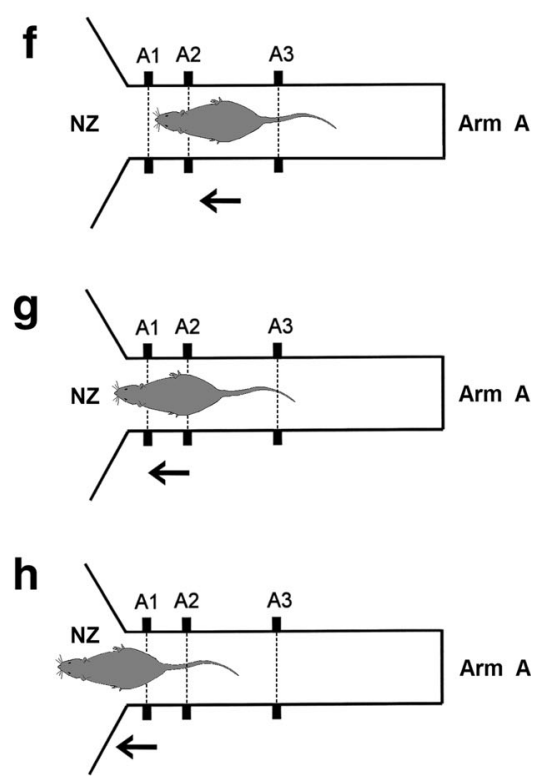

(d) shows a false entrance. Drawings (e) to (h) represent the sequence of optocoupler interruptions that corresponds to an arm exit 
Sequence of events for the detection of an entry into or an exit from an arm of the automated Y-maze

We adopted the arm entry criterion of Detrait et al. (2010), which for our system would be that the rat reaches past the middle third of the arm's length when moving into it. Two software algorithms were developed to detect the appropriate sequence of infrared beam interruptions to guarantee the faithful discrimination of the entries and exits from any arm, as exemplified for arm A in Fig. 2. When the rat enters the NZ (after exiting an arm) the system scans the status of the proximal OCs of all three arms. This status is obtained from the logic signal of the circuit associated with each receiver, which will change when an occlusion takes place.

Arm entry validation sequence When a proximal OC is blocked by a rat coming from the NZ, the medial and then the distal OCs of that same arm will be assessed. For instance, if the proximal beam of arm A (labeled as A1) is interrupted, its state will be monitored, along with the state of beam A2, which must be blocked after A1 in case the rat continues to advance into arm A (Fig. 2a). As long as these two beams remain occluded the arm entry is not yet validated. Finally, when A1 is no longer occluded but A3 becomes blocked (Fig. 2b), the arm entrance is recorded and the time count for the visit is initiated. Concurrently, the time count of the visit to the NZ prior to the entry to arm A ends and is saved in a non-volatile memory along with the maze zone identification (in this case the ASCII code for N, indicating the neutral zone). Figure $2 \mathrm{c}$ shows the rat no longer occluding beam $\mathrm{A} 3$, but before $\mathrm{A} 2$ or A1 have been blocked again. Thus, the rat is still considered to be inside arm A and, therefore, the time count for the visit continues.

False entry Figure $2 d$ shows an example of a false entry, in which the rat failed to complete the expected sequence of beam occlusions and non-occlusions that define a true entry.

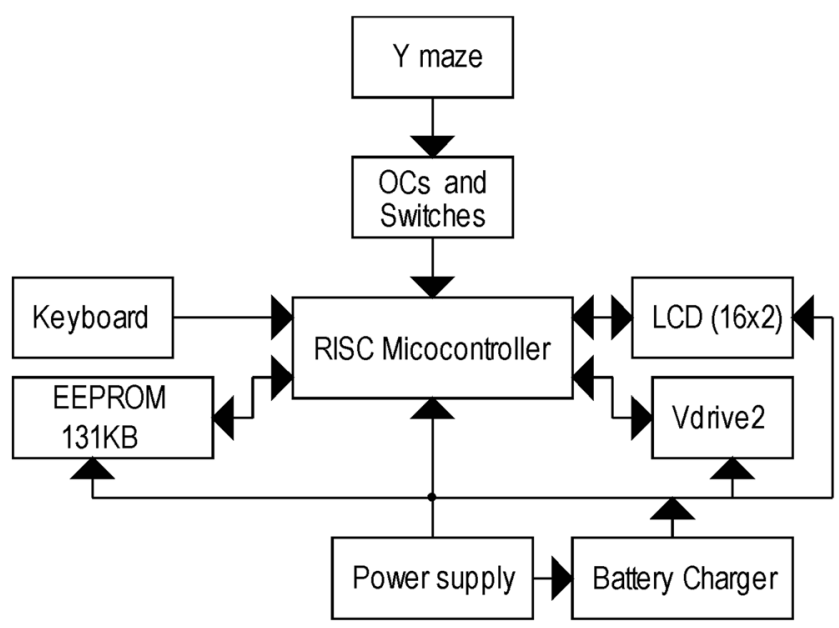

Fig. 3 Block diagram of the electronic control module
That is, the rat blocked $\mathrm{A} 1$ and then $\mathrm{A} 2$, but then turned around without occluding A3. This is not counted as an arm entry, regardless of whether the rat later exited the arm or not.

Arm exit validation sequence As stated before, the entry to arm $\mathrm{A}$ requires the occlusion of beam A3 (and the nonocclusion of beam A1). Figure 2e shows this situation with the rat facing the NZ. The exit sequence initiates when the rat moves towards the NZ, no longer interrupting beam $\mathrm{A} 3$, and eventually blocking beam A2 (Fig. 2f). Then all three OCs in the arm are monitored, first to detect that $\mathrm{A} 3$ remains uninterrupted and second to sense when $\mathrm{A} 1$ has been blocked, along with A2 (Fig. 2g). Finally, the arm visit ends when A2 is unblocked (Fig. $2 \mathrm{~h}$ ), and the resulting duration is stored. At the same time, a new visit to the NZ begins and the corresponding time count is started. When the programmed experimental time ends, the duration of the ongoing visit is truncated.

\section{Automated Y-maze hardware}

The microcontroller and the support circuitry are enclosed in a control module (Fig. 3), which is constructed around a PIC16F886 RISC eight-bit microcontroller (Microchip Inc., Chandler, AZ, USA), using its internal 8-MHz system clock. This microcontroller is programmed to read through its ports (A, B, and C) the digital signals coming from nine transistorized circuits that process the infrared signals sensed by the corresponding phototransistors mounted in the maze arms. These phototransistors are configured in an emitter follower configuration, and their output voltages drive the gates of $\mathrm{N}$ channel MOSFET transistors (type 2N7002, Fairchild Semiconductor Corp., San Jose, CA, USA) wired as logiclevel switches. This arrangement takes advantage of the lowthreshold gate voltage of the MOSFET devices that gives rise to a digital logic-level change (from 0 to $5 \mathrm{~V}$ ) at the output of a given switch whenever a light beam is obstructed by the rat.

A type FM24VN10 ferroelectric non-volatile RAM storage device (Ramtron International Corp., Colorado Springs, CO, USA) is used to save the data collected during the experiment. The total memory storage capacity is $128 \mathrm{kB}$. The software program allots a space of $2 \mathrm{kB}$ for the storage of each experiment, regardless of the number of arm visits performed by the rat. Thus, the capacity of the internal $128 \mathrm{kB}$ memory is enough to store up to 64 experiments.

A type LCR-01602DSF liquid crystal display (LCD) (Lumex Inc., Carol Stream, IL, USA) and a set of three pushbutton switches (which constitute a keyboard) are also handled by the microcontroller. The display allows the user to see which arm is currently occupied by the rat, while at the same time showing the letter of the arm visited previously, as well as the duration of that visit. The keyboard permits the user to introduce the experimentation time, to abort an 


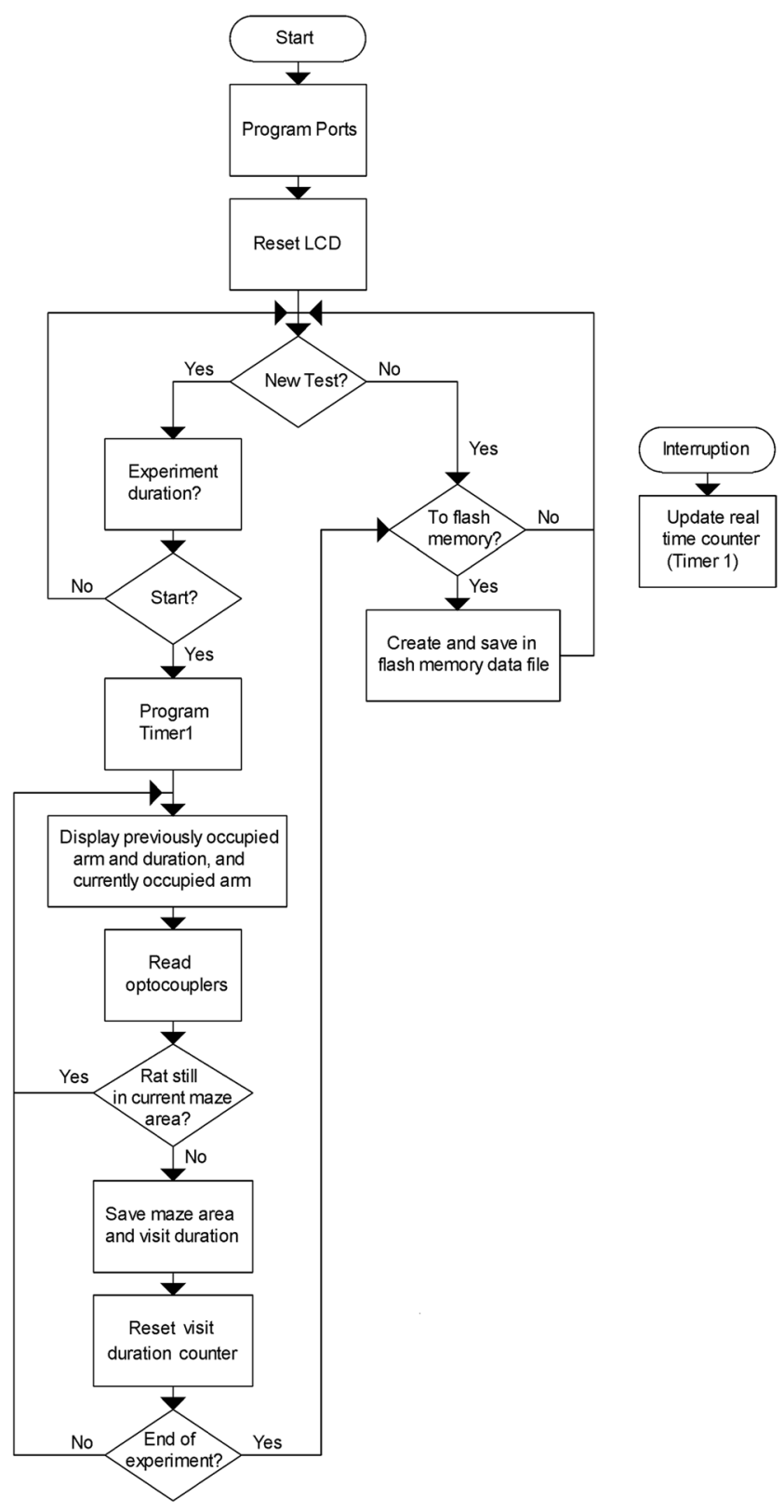

Fig. 4 Main software and interruption routine flow diagram

experiment, or to instruct that data be saved on an external flash USB memory. A Vdrive2 USB flash disk interface addon module (Future Technology Devices International Ltd., Tigard, OR, USA) is used to create an output text file in the USB flash memory attached to the USB connector of the control module. The name of the file is taken from the experimentation code selected by the user when a new experiment is started. Data in the FM24VN10 internal memory are recovered and processed to create the data file.

All the previously described circuits are powered by a $9-\mathrm{V}$ DC power supply that is down- regulated to $5 \mathrm{~V}$ using an uA78M05 linear voltage regulator (Texas Instruments Inc., Dallas, TX, USA). This 5-V source is used to supply energy via a $60-\mathrm{V} / 1$ A Schottky diode to a type LT1935 regulated boost charge pump (Linear Technology Corp., Milpitas, CA, USA) programmed to supply a regulated voltage of $5 \mathrm{~V}$, which is used to provide energy to the entire system. In addition, the 5-V supply feeds a type MCP73833 single-cell Li-ion charger (Microchip Inc., Chandler, AZ, USA), which recharges a 3.3-V/0.7 Ah Li-ion battery. This battery provides energy to the system during blackouts sending power through a second 60-V/1 A Schottky diode to the charge pump.

\section{Automated Y-maze software}

Assembly language firmware for the microcontroller was developed to instruct the microcontroller to scan the OCs in each arm, determine the rat position, save the data (arm entries and durations) in memory, display this experimental information on the LCD, keep track of the experimental time, and save the collected data as an ASCII (text) file in the non-volatile internal memory of the RISC microcontroller for later transfer to a USB external flash memory. A flow diagram of this program is shown in Fig. 4. The interruption routine is used to keep track of the programmed experimental time. (All of the schematics, printed board files, and software code related to the automated Y-maze here described are freely available from: https://drive.google.com/file/d/0B63 YLK5rNdUQIRuQ3hQSENGNnc/view?usp=sharing).

The data stored in the ASCII files are analyzed with a program developed in LabVIEW 10.0 (National Instruments Corp., Austin, TX, USA), which was designed to evaluate only those experiments in which the rat made a minimum of nine arm visits, including the starting arm, given that it is recognized that lower numbers of visits yield unreliable alternation percentages (Drew et al., 1973; Fraser et al., 1997; Hooper et al., 1996). This program allows calculating the latency (s) for exiting the starting arm (A), the number and sequence of arm entries, the number of alternations, the alternation percentage, and the duration of all visits to each arm (s). The number of entries into the arms of the Y-maze is often used as a measure of animal locomotion. However, double entries are usually not counted, resulting in an underestimation of the distance traveled by the rodent. For this reason, our analysis program included an option (referred to as locomotion) consisting of the sum of all arm entries, including reentries, but excluding the initial visit to the starting arm (A), which is not considered a real entry for this purpose, given that the experimenter placed the rat there.

The developed program first opens a dialog box prompting the user to browse and locate the folder containing the ASCII files to be analyzed. Then it performs the analysis set and exports the results into an Excel ${ }^{\circledR}$ spreadsheet file. 


\section{Experimental evaluation of the automated Y-maze}

The performance of the automated Y-maze was assessed by measuring the continuous SAB of 24 male Wistar rats (34 months old), which were housed in groups of three in polycarbonate cages (length $41.5 \mathrm{~cm}$, width $24 \mathrm{~cm}$, height $21 \mathrm{~cm}$ ). They were acclimatized for 1 week in a room with a controlled temperature $\left(23 \pm 1{ }^{\circ} \mathrm{C}\right)$ under 12:12 h light/dark cycles (lights on at 07:00). Food and water were available ad libitum. Animals weighed $314 \pm 2 \mathrm{~g}$ on average (range 295-331 g) on the day of SAB testing. At the end of experiments animals were euthanized with an overdose of pentobarbital $(100 \mathrm{mg} / \mathrm{kg})$.

This study was approved by the Institutional Bioethics Committee of the CIR-UADY, and all efforts were made to minimize animal discomfort in compliance with the Mexican regulations for the care and use of laboratory animals (NOM062-ZOO-1999), and the Guide for the Care and Use of Laboratory Animals (National Research Council of USA, 1996)

In order to evaluate the ability of the automated system to detect the effects of drugs that influence continuous SAB, animals were randomly assigned to one the following treatments: (a) Vehicle (VEH, distilled water, $1 \mathrm{ml} / \mathrm{kg}$ ), (b) caffeine (CAFF, $3 \mathrm{mg} / \mathrm{kg}$ ), (c) trihexyphenidyl (THP, $1 \mathrm{mg} / \mathrm{kg}$ ), or (d) CAFF + THP. To ensure that each animal had the same level of stress caused by handling, each received two consecutive subcutaneous injections (veh+veh, veh+drug, or drug+drug) $30 \mathrm{~min}$ before the Y-maze test, which lasted $8 \mathrm{~min}$. The experimentation room had an exhaust fan for continuous air renewal, and a fluorescent light provided an adequate illumination intensity $(\approx 140 \mathrm{~lx})$ for albino rats. Between tests, the inner walls and bottom of the maze were thoroughly wiped with $70 \%$ ethanol, and the excess was removed with paper towels, leaving a 5-min pause to allow for a complete evaporation of ethanol vapors before starting the next trial. The experiments were videotaped and later analyzed independently by two trained observers, who were blinded to the treatments. The efficacy and accuracy of the automated system was verified by comparing its output results with those manually calculated by the observers.

\section{Data analysis}

The alternation percentage was calculated by the automated system and by the observers according to the standard formula (Drew et al., 1973):

\section{Alternation percentage}

$$
=[(\text { Number of alternations }) /(\text { Total arm entries }-2)] \times 100
$$

An alternation is defined as consecutive entries into three distinct arms (e.g., ACB) in overlapping triplet sets. The placement of the rat into arm A (starting point) is considered as the first entry (Drew et al., 1973). When the animal reenters an arm two or more consecutive times, it is considered as a single entry (e.g., BCCA would be equivalent to BCA). For the estimation of locomotion, however, the initial placement of the rat in arm A is excluded, but re-entries are counted as separate entries. Therefore, the sequence BCCA would be counted as four entries.

\section{Statistical analysis}

All numerical results are expressed as mean \pm standard deviation (SD). In accordance with the policy of the Society for Neuroscience (2015) for conducting studies in laboratory animals recommending the use of "the minimum number required to obtain valid results," the original number of animals per treatment was set to six, which is the smallest reported group size that we found in a continuous SAB study (Stone et al., 1992). However, four animals were discarded because they did not perform the minimum of nine arm entries. Since the statistical analysis yielded significant results, five was the final number of rats in each treatment group. In order to assess the degree of agreement between the results yielded by the automated system and those produced by the two independent observers, the Bland-Altman method for concordance was used (Bland \& Altman, 1986). This approach is better suited to assess the degree of concordance between continuous variables obtained with two measuring techniques or made by two observers, unlike the commonly, but mistakenly used, Pearson's correlation coefficient, which does not provide such information (Zaki et al., 2012; for a detailed description of Bland-Altman graphs see Kwiecien et al., 2011). The D'Agostino and Pearson omnibus K2 normality test was used to confirm that the data sets of the differences followed a Gaussian distribution, which is a requirement for the Bland-Altman analysis. The average values of the alternation percentages and all the other variables that were directly measured or computed by the two independent observers (locomotion, latencies, and duration of visits to each of the four maze areas) were compared with those recorded by the automatic system. Additionally, within each variable, the effects of drug treatments on the values registered by the automated system and the averages of the two observers were assessed separately with one-way ANOVA, followed by Tukey's post-test. Finally, one-sample $t$-tests were performed in order to determine whether rats alternated above chance level by comparing their alternation percentages per treatment group against the reference value of $50 \%$ (Detrait et al., 2010; Hooper et al., 1996). All analyses were performed using GraphPad Prism Ver. 5.03 (GraphPad Software Inc., San Diego, CA, USA) and Microsoft Excel® version 2013. 


\begin{tabular}{|c|c|c|c|c|c|c|c|c|c|c|c|c|}
\hline & A & B & $\mathrm{C}$ & D & $E$ & $\mathrm{~F}$ & G & $\mathrm{H}$ & 1 & $\mathrm{j}$ & $\mathrm{K}$ & L \\
\hline 1 & Code & Date(M/D/Y) & Latency(s) & Entries(\#) & Sequence & Alternations(\#) & Alternation(\%) & Locomotion(\#) & $t A(s)$ & $\mathrm{tB}(\mathrm{s})$ & $\mathrm{tC}(\mathrm{s})$ & $\mathrm{tNZ}(\mathbf{s})$ \\
\hline 2 & A02 & $12 / 11 / 2013$ & 33 & 17 & ACBACABCBCABCBCAB & 10 & 66.7 & 16 & 165 & 80 & 122 & 113 \\
\hline 3 & A03 & $12 / 10 / 2013$ & 360 & 4 & ACBA & \#\# & \#\# & 3 & 404 & 49 & 3 & 24 \\
\hline 4 & A05 & $12 / 10 / 2013$ & 14 & 18 & ACBACACBACBACBACAB & 13 & 81.2 & 17 & 161 & 103 & 119 & 97 \\
\hline 5 & A06 & 01/21/2014 & 10 & 20 & АВСВАСВАСВАСВАСВСАСВ & 15 & 83.3 & 19 & 130 & 126 & 121 & 103 \\
\hline 6 & A07 & 01/21/2014 & 16 & 25 & ACBCACABCBCACABCABCACBCBA & 13 & 56.5 & 25 & 126 & 112 & 120 & 122 \\
\hline 7 & A08 & $01 / 21 / 2014$ & 24 & 19 & ABCBACBACBCBACBCABC & 13 & 76.5 & 19 & 114 & 110 & 140 & 116 \\
\hline
\end{tabular}

Fig. 5 Spreadsheet displaying the results and analysis of six experiments. The symbol \#\#\# indicates that the alternation percentage was not calculated because the rat did not make at least nine arm visits

\section{Results}

Figure 5 shows a sample of the SAB results in rats treated with the vehicle, as displayed in the spreadsheet generated by the program coded with LabVIEW, and exported as an xls file. In the first column of the data table are the codes for each rat, the second column shows the dates of the experiments, and the rest of the column headers correspond to each of the output variables. Each row contains the values calculated for each animal. The name of the spreadsheet file is the same as that of the directory where the ASCII data files were originally saved. It should be pointed out that the alternation percentage for rat A03 was not calculated because it did not perform the minimum of nine arm visits.

As described earlier, we defined that an arm entry occurred when a rat interrupted the distal OCs located $26 \mathrm{~cm}$ from the arm entrance, a distance that exceeds the average body length of an adult rat (see Fig. 2b). By using this last criterion, the spontaneous alternation percentage calculated by the automated system and by the combined values of the two blind

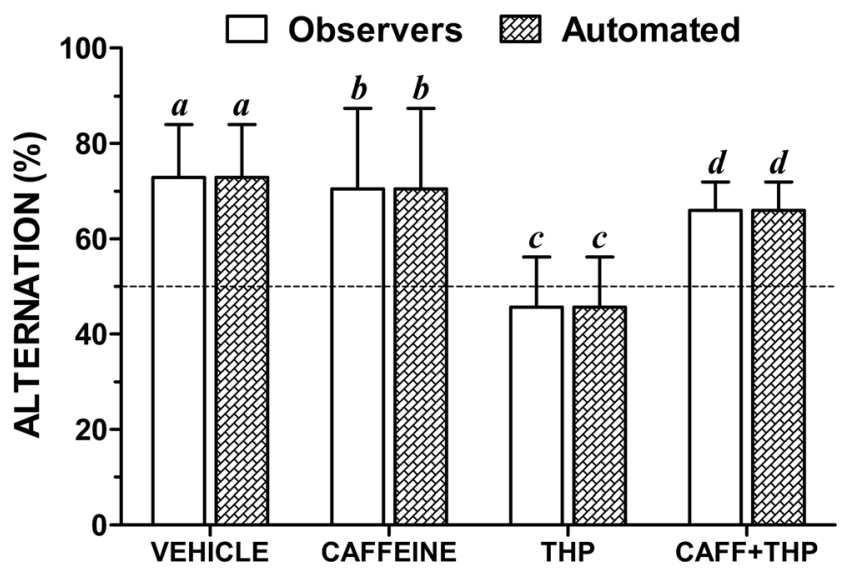

Fig. 6 Comparison of alternation percentages reported by the automated Y-maze and by the observers. Bars represent means \pm SDs ( $\mathrm{n}=5$ for all groups). Since the alternation percentages calculated by the two observers were $100 \%$ concordant, for simplicity their results were merged into a single bar for each treatment. Effect of treatments on alternation percentage: one-way ANOVA was run independently for the results of the automated system and for the average of the observer's values, but yielded identical results: $F_{(3,16)}=5.5, p=0.0087 ; \boldsymbol{a}$ vs. $\boldsymbol{c}$ and $\boldsymbol{b}$ vs. $\boldsymbol{c}, p<$ 0.05 (Tukey's post-test). Alternation percentage vs. $50 \%$ (random level, represented by the horizontal dashed line), one-sample $t$-test: $\boldsymbol{a}, t_{(4)}=$ $4.58, p=0.010 ; \boldsymbol{b}, t_{(4)}=2.70, p=0.054 ; \boldsymbol{c}, t_{(4)}=0.92, p=0.410 ; \boldsymbol{d}, t_{(4)}$ $=5.98, p=0.004$. THP trihexyphenidyl $1 \mathrm{mg} / \mathrm{kg}, C A F F$ caffeine $3 \mathrm{mg} / \mathrm{kg}$ observers had a concordance of $100 \%$ (Fig. 6). Similarly, both procedures yielded identical group average values in the number of valid alternations (distinct arm triplets) and entrances to the arms (Table 1), which are the two essential parameters for the calculation of alternation percentages.

When the effect of treatments on the variables of interest were independently analyzed with one-way ANOVA and the post hoc Tukey's test, in most cases the statistical results agreed between the two scoring methods, with the exception of time spent in arm B (Table 1). Thus, the values of locomotion, number of alternations, latencies, and time in arm $\mathrm{C}$ did not differ significantly between treatments, irrespective of the recording source. Analogously, within each scoring procedure the statistical analysis consistently detected a significant difference between caffeine- and THP-treated groups for arm A (superscript $a$ ) and the NZ (superscript $c$ ), and between vehicle and THP for the NZ (superscripts $d$ and $e$ ). On the other hand, a significant difference was observed between the caffeine and THP groups in the times spent in arm B as recorded by the automatic system (superscript $b$ ), but not in those counted by the two observers (Table 1).

The classical method for measuring SAB variables of rodents in a Y-maze is direct observation and manual counting. The largest disagreements between the values recorded by the automated system and by the observers occurred for the duration of visits to the arms and the NZ. Despite these discrepancies, Bland-Altman analysis showed good concordance between the duration of visits timed by the automated Y-maze and by the average of values computed by the two observers (Fig. 7).

The average of the differences between pairs of measurements \pm 1.96 times the SD is shown in Table 2. It can be seen that the two observers had $\geq 95 \%$ concordance in their calculated duration of visits to each of in the four maze areas. Among the four comparisons between values calculated by the automated system and the average of the two observers, three had a concordance of $95 \%$, while in only one it was $90 \%$ (time in arm B, Auto vs. AvgObs1+2). All the differences between pairs of values obtained by any two of the counting sources were normally distributed, except for the times spent in the NZ as counted by observers 1 and 2. Also, the discrepancies were greater for the duration of visits to the NZ, which is reflected in a wider region of concordance. 
Table 1 Comparison of Y-maze results between those reported by the automated system and the average values recorded by two independent trained observers

\begin{tabular}{|c|c|c|c|c|}
\hline Parameter & Treatment & Automated & {$[\mathrm{Obs} 1+\mathrm{Obs} 2] / 2$} & Percent error \\
\hline \multirow[t]{4}{*}{ Locomotion (n) } & Veh & $19.2 \pm 3.5$ & $19.2 \pm 3.5$ & 0 \\
\hline & Caff & $16.6 \pm 2.6$ & $16.6 \pm 2.6$ & 0 \\
\hline & THP & $17.4 \pm 5.0$ & $17.4 \pm 5.0$ & 0 \\
\hline & Caff+THP & $22.0 \pm 6.2$ & $22.0 \pm 6.2$ & 0 \\
\hline \multirow[t]{4}{*}{ Alternations (n) } & Veh & $12.8 \pm 1.8$ & $12.8 \pm 1.8$ & 0 \\
\hline & Caff & $10.4 \pm 1.1$ & $10.4 \pm 1.1$ & 0 \\
\hline & THP & $7.8 \pm 3.6$ & $7.8 \pm 3.6$ & 0 \\
\hline & Caff+THP & $13.8 \pm 5.4$ & $13.8 \pm 5.4$ & 0 \\
\hline \multirow[t]{4}{*}{ Latency (s) } & Veh & $19.4 \pm 9.2$ & $19.4 \pm 9.2$ & 0 \\
\hline & Caff & $27.6 \pm 21.9$ & $27.5 \pm 21.9$ & 0.4 \\
\hline & THP & $24.4 \pm 10.6$ & $24.7 \pm 11.1$ & -1.2 \\
\hline & Caff+THP & $21.6 \pm 13.0$ & $21.2 \pm 12.9$ & 1.9 \\
\hline \multirow[t]{4}{*}{ Time in arm A (s) } & Veh & $139.2 \pm 22.6$ & $144.5 \pm 22.7$ & -3.7 \\
\hline & Caff & $151.0 \pm 14.1^{a}$ & $157.7 \pm 13.0^{a}$ & -4.3 \\
\hline & THP & $89.0 \pm 45.7^{a}$ & $92.6 \pm 47.1^{a}$ & -4.0 \\
\hline & Caff+THP & $141.6 \pm 32.8$ & $145.0 \pm 33.2$ & -2.4 \\
\hline \multirow[t]{4}{*}{ Time in arm B (s) } & Veh & $106.2 \pm 16.9$ & $107.2 \pm 18.6$ & -0.9 \\
\hline & Caff & $102.0 \pm 25.0^{b}$ & $101.9 \pm 25.6$ & 0.1 \\
\hline & THP & $159.2 \pm 51.4^{b}$ & $157.7 \pm 51.9$ & 0.9 \\
\hline & Caff+THP & $111.2 \pm 18.0$ & $109.8 \pm 18.6$ & 1.3 \\
\hline \multirow[t]{4}{*}{ Time in arm C (s) } & Veh & $124.4 \pm 8.8$ & $124.9 \pm 9.9$ & -0.4 \\
\hline & Caff & $121.6 \pm 31.4$ & $122.6 \pm 31.1$ & -0.8 \\
\hline & THP & $174.0 \pm 65.9$ & $172.7 \pm 66.0$ & 0.7 \\
\hline & Caff+THP & $150.6 \pm 50.2$ & $150.0 \pm 49.3$ & 0.4 \\
\hline \multirow[t]{4}{*}{ Time in the NZ (s) } & Veh & $110.2 \pm 10.1^{e}$ & $105.4 \pm 12.5^{d}$ & 4.5 \\
\hline & Caff & $105.4 \pm 40.0^{c}$ & $101.0 \pm 37.5^{c}$ & 4.3 \\
\hline & THP & $57.8 \pm 16.0^{c, e}$ & $59.7 \pm 13.4^{c, d}$ & -3.2 \\
\hline & Caff+THP & $76.6 \pm 8.2$ & $78.3 \pm 8.3$ & -2.2 \\
\hline
\end{tabular}

Values are the means \pm SDs of five rats per treatment, except for the last column, which contains the percent errors (or differences) between the automated system results (third column) and the average of the results reported by the two observers (fourth column) \% error $=\{($ Auto-Avg. Obs $) /[$ Auto + Avg. Obs $) /$ 2] $\} \times 100$

Values in the same column marked with the same superscript letter are significantly different. $\boldsymbol{a}, \boldsymbol{b}, \boldsymbol{c}, \boldsymbol{d}, p<0.05 ; \boldsymbol{e}, p<0.01$ (Tukey's post-test; see explanation in text). Note that these differences are due to the applied treatments and not to the measurement techniques

Obs observer, Veh vehicle, Caff caffeine, THP trihexyphenidyl, NZ neutral zone

\section{Discussion}

The results of the experimental evaluation indicate that the automated Y-maze based on infrared beams governed by a RISC microcontroller is completely reliable, since it registers the sequence of real entries, ignoring false entries or exits and, therefore, it has a good concordance with the average of the results computed by two trained, independent observers after analyzing videos of the same SAB trials in rats. This was achieved by designing two software algorithms (one for detecting arm entrances, and another for exits) that examine the interruption sequence of infrared beams emitted by three pairs of OCs, the spacings of which were chosen to optimally detect that the entire body length of an adult rat was already located in the medial or distal thirds of each arm, thus ensuring that the animal had performed a true entry, according to the criterion reported by Detrait et al. (2010). In addition, the software algorithm that analyzes arm exits was designed to prevent the recording of spurious double entries when the animal walks towards the exit of the arm and, without entering the $\mathrm{NZ}$, turns back into the arm and again interrupts the distal infrared beam, as was reported by Lennartz (2008) in the evaluation of a plus maze whose arms were also equipped with three infrared beams. However, in that study the sensors were located at $5.1,7.3$, and $15.5 \mathrm{~cm}$ from the NZ, and at a height of $3.8 \mathrm{~cm}$ from the maze floor. With this spacing of the 


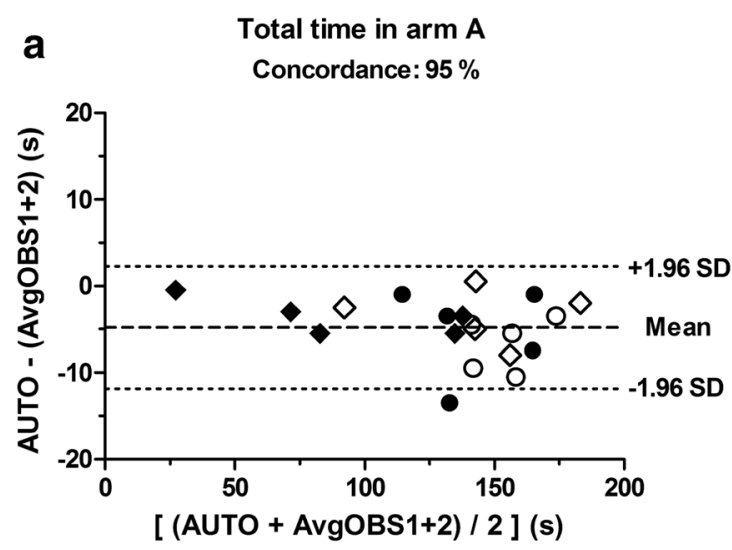

C

Total time in arm C

Concordance: $95 \%$

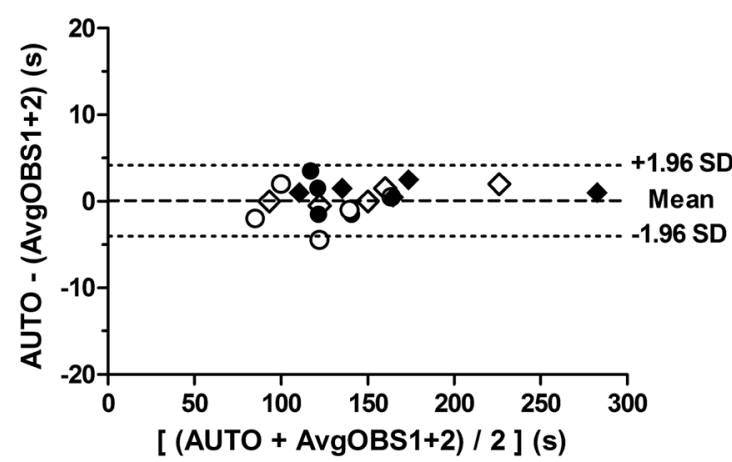

Fig. 7 Bland-Altman graphs to assess the concordance of time values for visits to each four maze areas, as recorded by the automated system (AUTO) and the average of values computed by the two observers $($ AvgOBS1+2). Treatments applied to rats are indicated by symbols: $(\bullet)$ vehicle, $(O)$ caffeine, $(\diamond)$ THP, and $(\diamond)$ caffeine + THP $(\mathrm{n}=5$ for all groups). The average of measurements taken by a couple of procedures (or subjects) is plotted in the abscissa, and the difference between them in the ordinate. The dashed line indicates the average of the differences

sensors, the entrances to the arms were recorded using a commercial software (MED-PC Version IV; Med Associates,

\section{b Total time in arm B \\ Concordance: $90 \%$}
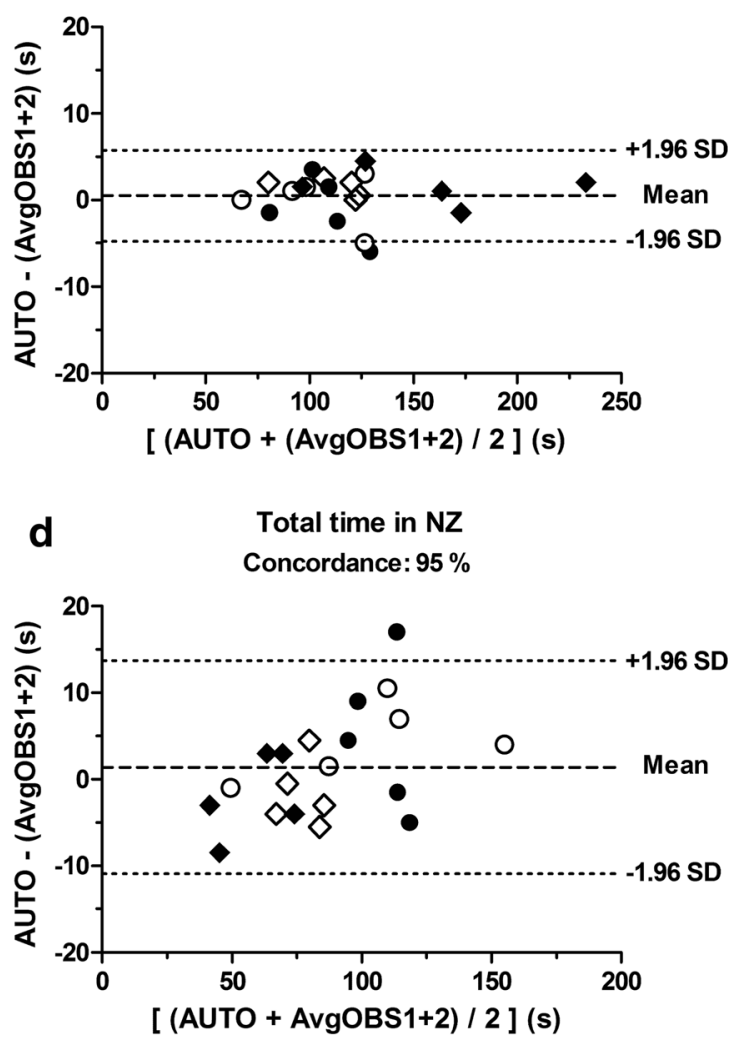

between every pair of measurements, and the dotted lines indicate \pm 1.96 times the standard deviation (SD) of these differences away from the mean, which mark the limits of concordance. Values above the upper limit $(+1.96 \mathrm{SD})$ or below the lower limit $(-1.96 \mathrm{SD})$ are considered not concordant. In the comparisons of the time data sets for arms $\mathrm{A}$ and $\mathrm{C}$ and the NZ only one out of 20 values was outside the limits, indicating a concordance of $95 \%$. For arm B, the concordance was $90 \%$ (two out of 20 values outside the range)

Georgia, VT, USA), but the data collection settings were not provided, and the alternation percentages were the only

Table 2 Bland-Altman concordance analysis of time values recorded by the automated system (Auto) and the average of values computed by the two observers (AvgObs1+2)

\begin{tabular}{|c|c|c|c|c|}
\hline Arm & Comparison & $\begin{array}{l}\text { Mean } \pm[1.96 \times \mathrm{SD}] * \\
(\mathrm{~s})\end{array}$ & Normality & Concordance (\%) \\
\hline \multirow[t]{2}{*}{ A } & Obs1 vs. Obs2 & $-4.7 \pm 9.0$ & Yes & 95 \\
\hline & Auto vs. AvgObs1+2 & $-4.8 \pm 7.1$ & Yes & 95 \\
\hline \multirow[t]{2}{*}{ B } & Obs1 vs. Obs2 & $-2.3 \pm 6.7$ & Yes & 95 \\
\hline & Auto vs. AvgObs1+2 & $0.5 \pm 5.3$ & Yes & 90 \\
\hline \multirow[t]{2}{*}{$\mathrm{C}$} & Obs1 vs. Obs2 & $-1.4 \pm 7.1$ & Yes & 100 \\
\hline & Auto vs. AvgObs1+2 & $0.1 \pm 4.1$ & Yes & 95 \\
\hline \multirow[t]{2}{*}{$\mathrm{N}$} & Obs1 vs. Obs2 & $3.3 \pm 19.4$ & No & 95 \\
\hline & Auto vs. AvgObs1+2 & $1.4 \pm 12.3$ & Yes & 95 \\
\hline
\end{tabular}

*Averages $\pm[1.96 \times \mathrm{SD}]$ of the differences in seconds computed by the two counting sources indicated in each case. Pooled data from 20 experiments (five rats in each of the four treatment groups). The width of the region of concordance may be calculated by subtracting the value of the mean minus $[1.96 \times \mathrm{SD}]$ from the mean plus $[1.96 \times \mathrm{SD}]$ 
reported data. The author commented that the automated plusmaze occasionally recorded false entries or consecutive reentries into the same arm, which were excluded from the analysis of alternation by inspecting videos of the SAB trials (Lennartz, 2008). Given the scarcity of information about the hardware and software of the automated plus-maze it is not possible to make a fair comparison with the performance of the automated Y-maze described here.

The efficiency of this new automated Y-maze was verified by its ability to measure the changes in spontaneous alternation percentages induced pharmacologically in rodents. Thus, while the vehicle-treated animals had an alternation percentage that was significantly higher than $50 \%$ (chance level), those treated with the muscarinic antagonist THP decreased their alternation percentages to values close to or lower than $50 \%$, which on average were significantly lower than those of the control group. The above results are in agreement with numerous reports showing that scopolamine, another muscarinic antagonist, interferes with working memory since it reduces the alternation percentage to values that do not differ significantly from $50 \%$, and which are significantly lower than those obtained for controls (Detrait et al., 2010; Drew et al., 1973; Fraser et al., 1997; Hefco et al., 2003; Hooper et al., 1996; Lelong et al., 2003; Sarter et al., 1988; Stone et al., 1992). In addition, the automated Y-maze described here was able to accurately measure the reversal of the amnesic effect of THP when it was co-administered with a low dose of caffeine, a non-selective adenosine receptor antagonist that is capable of preventing the short-term memory impairment caused by scopolamine in mice (Botton et al., 2010). The results obtained with the automated Y-maze also showed that caffeine alone was ineffective at increasing the alternation percentage, in comparison with controls, a finding that is in line with the notion that caffeine is not a cognitive enhancer (Cunha \& Agostinho, 2010).

It is important to note that an initial inspection of the numerical data showed some discrepancies between the percentages of alternation calculated by one of the observers and those obtained with the automated maze. This led to repeating the manual analysis, and it was found that the inconsistencies were due either to transcription errors or to manual miscalculations of the alternation percentage. After making the pertinent corrections the results were identical to those reported by the automatic system.

The largest disparities between measurements taken by the automated system and by the observers occurred in the duration of visits to the arms and the NZ. These discrepancies also occurred between the two observers, which is explained by the great difficulty in counting these intervals by visual inspection, probably because often the rats moved quickly from one area to another. Since the total time spent in each of the maze areas is calculated by adding the times per visit, and each measurement involves the possibility of a counting error on the part of the observers, it is plausible that their sums could yield larger differences compared with those of the automated system. Despite this, the Bland-Altman analysis, which provides a graphical and quantitative representation of the closeness between each pair of values being compared (Zaki et al., 2012), revealed that the duration of visits counted by the automated system and by the averages of the values calculated by the two observers had a concordance equal or greater than $95 \%$ for most comparisons (Fig. 7 and Table 1), supporting the idea that the electronic device reported here is as reliable as, and probably more precise than, a trained experimenter. In this respect it should be noted that the visit durations to arm B as reported by the automated maze were significantly different between the rats treated with caffeine and those that received THP. However, this difference was not significant when the statistical analysis was performed with the values obtained manually for both groups of rats, despite their similarity to the ones reported by the automated system (Table 1), possibly because the small counting errors made by the observers were enough to mask a true difference at the 0.05 significance level, and considering the reduced sample size that was used. It is worth mentioning that out of the 28 comparisons made for the seven Y-maze parameters in the four experimental groups, all the percent differences were under $5 \%$ (Table 1), and most of them were even under $3 \%$.

Studies based on the continuous SAB test have used Ymazes with radial symmetry (e.g., three arms of equal length, separated by $120^{\circ}$ angles). In contrast, the automated system described here is a Y-maze with bilateral symmetry, with two arms (B and $\mathrm{C}$ ) of equal length and separated at an angle of $156^{\circ}$, and a third arm (A), which is $4 \mathrm{~cm}$ longer and is separated from the other two by $102^{\circ}$ angles. These structural characteristics were due to the fact that this Y-maze was originally devised to perform an object recognition test manually. However, it is unlikely that the lack of radial symmetry of the automated Y-maze had biased the results since the average alternation percentage of control rats $(73 \%)$ is within the range of values $(68-75 \%)$ reported in other studies that evaluated continuous SAB of rats in Y-mazes with arms symmetrically spaced at $120^{\circ}$ angles (Drew et al., 1973; Hefco et al., 2003; Krebs-Kraft \& Parent, 2008; Stefani et al., 1999). This makes us confident that the hardware and software described in this paper would work with the same efficiency in Y-mazes with arms of different lengths and separation angles.

Another possible application of the automated Y-maze is the novel object recognition (NOR) test which is used to study recognition memory, and relies on the animal's natural preference for novelty (Antunes \& Biala, 2012). In its original form this test is executed in two stages (Dellu et al., 1992). Initially, two identical objects are placed in arms B and C of a Y-maze, and then the rodent is introduced in arm A, allowing it to freely inspect the maze during a fixed period of time. In a second trial, following a specific time interval, one of the 
objects is replaced with a different one, and then the animal is reintroduced in arm A. The time that it spends exploring each maze arms is counted. Normal animals usually devote more time to exploring the arm where the novel stimulus (new object) is located, a measure that is considered a reflection of the recognition memory of the animal (Antunes \& Biala, 2012; Dellu et al., 1992). In the original description of the NOR test, Dellu et al. (1992) used a Y-maze with two infrared beams situated at 3 and $25 \mathrm{~cm}$ from each arm entrance, whose sequential interruption was required to record an arm entry, although details of the hardware and software of the system were not provided. More recently the automation of the NOR test in a Y-maze using a commercial video-tracking system (EthoVision XT®) to monitor the duration of visits to the distal halves of the arms where the objects were placed was reported (Chambon et al., 2011). Since results showed that the parameter that best reflected novel object discrimination was the time spent by rats in the distal half of the arms (Chambon et al., 2011), we suggest that the automated system described here could also be used for the NOR test by simply increasing the length and width of each arm in order to allow the rat to explore around the object (Chambon et al., 2011).

The automated Y-maze described here was intended for measuring the variables of continuous $\mathrm{SAB}$ in adult rats, whose body weight and length do not exceed $400 \mathrm{~g}$ and $26 \mathrm{~cm}$, respectively. Hence, since the positions of the OCs are not adjustable, it has the possible limitation of yielding inaccurate $\mathrm{SAB}$ measurements for animals with body lengths greater than $26 \mathrm{~cm}$. Indeed, it has been reported that male Wistar rats can attain an average body length of $28.2 \pm$ $0.9 \mathrm{~cm}(\mathrm{SD}, \mathrm{n}=14)$ when they reach a weight of $593 \pm 65 \mathrm{~g}$ (Magnani et al., 2012). Despite the last possibility, it should be noted that the studies of continuous SAB in adult rats are usually performed in animals weighing no more than $300 \mathrm{~g}$ (Drew et al., 1973; Hefco et al., 2003; Krebs-Kraft \& Parent, 2008; Stefani et al., 1999), presumably with a body length of less than $26 \mathrm{~cm}$.

With regard to mice, average alternation values are in the range of $68-74 \%$ when they are evaluated in Y-mazes with arms $22 \mathrm{~cm}$ long and $6.5 \mathrm{~cm}$ wide (Fraser et al., 1997; Lelong et al., 2003), which is not much different to the value of $\approx 70 \%$ recorded in mice tested in a maze with arms $60 \mathrm{~cm}$ long and $3.5 \mathrm{~cm}$ wide (Stone et al., 1992). This indicates that the percentage values of spontaneous alternation in mice are very similar to those recorded in rats, regardless of the length and width of the arms, which in the case of this automated Y-maze are 46 and $14.6 \mathrm{~cm}$, respectively. Considering that the body length of adult male mice can attain on average $10.3 \mathrm{~cm}(\mathrm{n}=310$; Castle, 1938), it is likely that the automated Y-maze could work well for mice by lowering the height of the sensors to $2.5 \mathrm{~cm}$, and relocating the position of the proximal, medial, and distal OCs at 2, 5, and $12 \mathrm{~cm}$, respectively, from the NZ.

\section{Conclusion}

The experimental validation of the automated Y-maze described here indicates that it is capable of recording the continuous SAB of rats with more accuracy and reliability than a well-trained observer, since the manual data acquisition task is subject to errors due to fatigue or distractions, as well as to transcription mistakes. Its advantages include: (1) it is a low cost device that can be reproduced easily since the readily available electronic components of a single system do not cost more than US\$100; (2) it is a stand-alone system that does not require a permanent connection to a computer or a video camera to detect and store the collected data; (3) the experimental data are permanently saved as ASCII files in a non-volatile memory until the experimenter decides to transfer or erase them; (4) the large storage capability of the internal memory allows the execution of many tests (up to 64), and at the beginning of each experiment the system displays how much space is left for data storage; (5) the backup battery, which can be charged while running the experiments, allows recording trials for up to $4 \mathrm{~h}$ without any data loss in case of power failure; (6) the use of a Vdrive2 module allows the transfer of the experimental data to any standard flash memory, and (7) the program developed in LabVIEW® allows a fast, error-free data analysis of single or sets of data files.

Acknowledgments This work was supported by grants no. 47763 (J.L. G.-A) and no. LN232719 (F.J. H.-L.) from the Consejo Nacional de Ciencia y Tecnología de México, and grant no. 2009-01-113 (F.J. H.L.) from Fondo Sectorial Salud, México.

Author contributions F.J. H.-L., F.J. A.-C., and J.G. C.-A. contributed equally to this work. They were responsible for the design of the electronics and software described here. Both F.J. H.-L. and F.J. A.-C. made major contributions to manuscript writing and editing. J.G. C.-A. and J.L. B.-G. performed the behavioral experiments. G. A.-S. performed statistical analyses and contributed to the manuscript writing. J.L. G.-A analyzed and interpreted drug-induced behavioral results, and made major contributions to the writing and editing of the paper.

\section{Compliance with ethical standards}

Competing interests The authors declare that they have no competing interests.

\section{References}

Antunes, M., \& Biala, G. (2012). The novel object recognition memory: Neurobiology, test procedure, and its modifications. Cognitive Processing, 13, 93-110. doi:10.1007/s10339-011-0430-z

Bailoo, J. D., Bohlen, M. O., \& Wahlsten, D. (2010). The precision of video and photocell tracking systems and the elimination of tracking errors with infrared backlighting. Journal of Neuroscience Methods, 188, 45-52. doi:10.1016/j.jneumeth.2010.01.035 
Bland, J.M., \& Altman, D.G. (1986). Statistical methods for assessing agreement between two methods of clinical measurement. Lancet, 327 (Iss. 8476), 307-310. doi:10.1016/S0140-6736(86)90837-8

Botton, P. H., Costa, M. S., Ardais, A. P., Mioranzza, S., Souza, D. O., da Rocha, J. B., \& Porciúncula, L. O. (2010). Caffeine prevents disruption of memory consolidation in the inhibitory avoidance and novel object recognition tasks by scopolamine in adult mice. Behavioural Brain Research, 214, 254-259. doi:10.1016/j.bbr.2010.05.034

Castle, W. E. (1938). The relation of albinism to body size in mice. Genetics, 23, 269-274.

Chambon, C., Wegener, N., Gravius, A., \& Danysz, W. (2011). A new automated method to assess the rat recognition memory: Validation of method. Behavioural Brain Research, 222, 151-157. doi:10. 1016/j.bbr.2011.03.032

Cunha, R. A., \& Agostinho, P. M. (2010). Chronic caffeine consumption prevents memory disturbance in different animal models of memory decline. Journal of Alzheimer's Disease, 20(Suppl 1), S95-S116. doi:10.3233/JAD-2010-1408

Dellu, F., Mayo, W., Cherkaoui, J., Le Moal, M., \& Simon, H. (1992). A two-trial memory task with automated recording: Study in young and aged rats. Brain Research, 588, 132-139. doi:10.1016/00068993(92)91352-F

Detrait, E., Brohez, C., Hanon, E., \& Ryck, M. (2010). Automation of continuous spontaneous alternation to increase the throughput for in vivo screening of cognitive enhancers. Optimization of the Ethovision system for the Y-maze test in mice. In: A. J. Spink, F. Grieco, O. E. Krips, L. W. S. Loijens, L. P. J. J. Noldus, \& P. H. Zimmerman (Eds.). Proceedings of Measuring Behavior 2010 (Eindhoven, The Netherlands, August 24-27, 2010) (pp. 141-144).

Drew, W. G., Miller, L. L., \& Baugh, E. L. (1973). Effects of $\Delta^{9}$-THC, LSD-25 and scopolamine on continuous, spontaneous alternation in the Y-maze. Psychopharmacologia (Berl), 132, 171-182. doi:10. 1007/BF00428688

Fraser, C. M., Fisher, A., Cooke, M. J., Thompson, I. D., \& Stone, T. W. (1997). Purine modulation of dizocilpine effects on spontaneous alternation. Psychopharmacology, 130, 334-342. doi:10.1007/ s002130050248

Hefco, V., Yamada, K., Hefco, A., Hritcu, L., Tiron, A., Olariu, A., \& Nabeshima, T. (2003). Effects of nicotine on memory impairment induced by blockade of muscarinic, nicotinic and dopamine D2 receptors in rats. European Journal of Pharmacology, 474, 227232. doi:10.1016/S0014-2999(03)02034-X

Hooper, N., Fraser, C., \& Stone, T. W. (1996). Effects of purine analogues on spontaneous alternation in mice. Psychopharmacology, 123, 250-257. doi:10.1007/BF02246579

Hughes, R. N. (2004). The value of spontaneous alternation behavior $(\mathrm{SAB})$ as a test of retention in pharmacological investigations of memory. Neuroscience \& Biobehavioral Reviews, 28, 497-505. doi:10.1016/j.neubiorev.2004.06.006

Krebs-Kraft, D. L., Parent, M. B. (2008). Hippocampal infusions of glucose reverse memory deficits produced by co-infusions of a GABA receptor agonist. Neurobiology of Learning \& Memory, 89, 142152. doi:10.1016/j.nlm.2007.07.008

Kwiecien, R., Kopp-Schneider, A., \& Blettner, M. (2011). Concordance analysis - part 16 of a series on evaluation of scientific publications. Deutsches Ärzteblatt International, 108, 515-521. doi:10.3238/ arztebl.2011.0515
Lalonde, R. (2002). The neurobiological basis of spontaneous alternation. Neuroscience \& Biobehavioral Reviews, 26, 91-104. doi:10.1016 S0149-7634(01)00041-0

Lelong, V., Lhonneur, L., Dauphin, F., \& Boulouard, M. (2003). BIMU 1 and RS 67333, two 5- $\mathrm{HT}_{4}$ receptor agonists, modulate spontaneous alternation deficits induced by scopolamine in the mouse. NaunynSchmiedeberg's Archives of Pharmacology, 367, 621-628. doi:10. 1007/s00210-003-0743-2

Lennartz, R. (2008). The role of extramaze cues in spontaneous alternation in a plus-maze. Learning \& Behavior, 36, 138-144. doi:10. 3758/LB.36.2.138

Magnani, K. L., Cataneo, D. C., Capelozzi, V. L., Defaveri, J., Hasimoto, E. N., \& Cataneo, A. J. (2012). Lung morphology and growth of rats exposed to tobacco smoke and alcohol. Acta Cirúrgica Brasileira, 27, 687-693. doi:10.1590/S0102-86502012001000004

McCay, C. M., Crowell, M. F., \& Maynard, L. A. (1935). The effect of retarded growth upon the length of life span and upon the ultimate body size. The Journal of Nutrition, 10, 63-79.

McLelland, A. E., Winkler, C. E., \& Martin-Iverson, M. T. (2015). A simple and effective method for building inexpensive infrared equipment used to monitor animal locomotion. Journal of Neuroscience Methods, 243, 1-7. doi:10.1016/j.jneumeth.2015.01. 006

Morellini, F. (2013). Spatial memory tasks in rodents: What do they model? Cell and Tissue Research, 354, 273-286. doi:10.1007/ s00441-013-1668-9

National Research Council of USA. (1996). Guide for the care and use of laboratory animals. Washington: National Academy Press.

Pullen, A. H. (1976). A parametric analysis of the growing CFHB (Wistar) rat. Journal of Anatomy, 121(Pt 2), 371-383.

Sarter, M., Bodewitz, G., \& Stephens, D. N. (1988). Attenuation of scopolamine-induced impairment of spontaneous alternation behaviour by antagonist but not inverse agonist and agonist $\beta$-carbolines. Psychopharmacology, 94, 491-495. doi:10.1007/BF00212843

Society for Neuroscience. (2015). Policies on the Use of Animals and Humans in Research, http://www.sfn.org/Advocacy/PolicyPositions/Policies-on-the-Use-of-Animals-and-Humans-inResearch (last consulted: 01/30/15).

Stefani, M. R., Nicholson, G. M., Gold, P. E. (1999). ATP-sensitive potassium channel blockade enhances spontaneous alternation performance in the rat: a potential mechanism for glucose-mediated memory enhancement. Neuroscience, 93, 557-563. doi:10.1016/S03064522(99)00128-1

Stone, W. S., Rudd, R. J., \& Gold, P. E. (1992). Glucose attenuation of scopolamine- and age-induced deficits in spontaneous alternation behavior and regional brain $\left[{ }^{3} \mathrm{H}\right] 2$-deoxyglucose uptake in mice. Psychobiology, 20, 270-279. doi:10.3758/BF03332059

Yamada,K., Hiramatsu, M., Noda, Y., Mamiya, T., Murai, M., Kameyama, ... Nabeshima, T. (1996). Role of NO and cyclic GMP in the dizocilpine-induced impairment of spontaneous alternation behavior in mice. Neuroscience, 74, 365-374. doi:10.1016/ 0306-4522(96)00161-3

Zaki, R., Bulgiba, A., Ismail, R., \& Ismail, N. A. (2012). Statistical methods used to test for agreement of medical instruments measuring continuous variables in method comparison studies: A systematic review. PLoS One, 7, e37908. doi:10.1371/journal.pone. 0037908 